\title{
DSP Based Series-Parallel Connected Two Full-Bridge DC-DC Converter with Interleaving Output Current Sharing
}

\author{
Deshang Sha ${ }^{\dagger}$, Zhiqiang Guo*, and Xiaozhong Liao* \\ ${ }^{*}$ School of Automation, Beijing Institute of Technology, Beijing, China
}

\begin{abstract}
Input-series-output-parallel (ISOP) connected DC-DC converters enable low voltage rating switches to be used in high voltage input applications. In this paper, a DSP is adopted to generate digital phase-shifted PWM signals and to fulfill the closed-loop control function for ISOP connected two full-bridge DC-DC converters. Moreover, a stable output current sharing control strategy is proposed for the system, with which equal sharing of the input voltage and the load current can be achieved without any input voltage control loops. Based on small signal analysis with the state space average method, a loop gain design with the proposed scheme is made. Compared with the conventional IVS scheme, the proposed strategy leads to simplification of the output voltage regulator design and better static and dynamic responses. The effectiveness of the proposed control strategy is verified by the simulation and experimental results of an ISOP system made up of two full-bridge DC-DC converters.
\end{abstract}

Key Words: Current sharing, Digital control, Input-series and output-parallel, Modular DC/DC converters, Phase-shifted fullbridge, Voltage sharing

\section{INTRODUCTION}

A phase-shifted full-bridge (PSFB) DC-DC converter permits all switching devices to operate under zero-voltage switching (ZVS) by using parasitic parameters such as leakage inductance and junction capacitance to achieve resonant switching. But under high input voltages, the switches have to withstand high voltage stress with the conventional PSfull-bridge StateDC-DC topologies. Three-level FB PS-PWM DC-DC converters enable low-voltage rating switches to be used under high-voltage input applications. However, system reliability can not be guaranteed for a large quantity of diodes or flying capacitors [1],[2].

The input-series output-parallel (ISOP) configuration consists of several modular DC-DC converters connected in series at the input and in parallel at the output, enabling the use of high switching frequency metal oxide semiconductor field effect transistors (MOSFETs) with low voltage ratings, which leads to a high power density and a high conversion efficiency [3]. Besides, modular architecture has the advantage of redundant operation capability [4], and therefore, the overall reliability is improved.

Generally, analog PWM controllers are used to implement the control of ISOP connected FB-PS DC-DC converters. However, analog control circuits are complex and lack flexi-

\footnotetext{
Manuscript received Mar. 12, 2010; revised Jun. 14, 2010

$\dagger$ Corresponding Author: shadeshang@bit.edu.cn

Tel: +86-10-68912460, Beijing Institute of Technology

* School of Automation, Beijing Institute of Technology, China
}

bility. These PWM controllers sometimes need to be synchronized to generate interleaving duty cycles. Besides, the cost of phase shifted PWM controllers is less competitive than digital controllers because each module needs one single controller. Although a digital PS PWM control strategy has been used for one full-bridge converters [5], digital PS PWM generation and control of ISOP connected modules has not been discussed.

To make an ISOP system work normally, the power sharing among the constituent modules should be ensured, and this also implies input voltage sharing (IVS) and output current sharing (OCS). Several control schemes have been proposed in earlier works. Common duty ratio control results in stable operation, but excellent IVS and OCS can only be achieved for modules with well-matched parameters [6],[7]. A charge control scheme with input-voltage feed forward has been implemented for a two-converter ISOP system [3]. However, input currents as well as input voltages have to been sensed. A three loop control scheme is implemented by sensing both the input voltages and the output currents [8],[9]. However, from the point view of power balance, achieving IVS can automatically realize OCS. As a result, it is unnecessary to implement both IVS and OCS control. A decoupling IVS control scheme [10]-[12], master/slave control [13] and uniform input voltage distribution control [14] for ISOP converters have been implemented with IVS loops without sensing the output currents at all. Sensorless current mode control is effective for an ISOP system [16], but component tolerances will lead to unbalanced voltage sharing among the modules. 


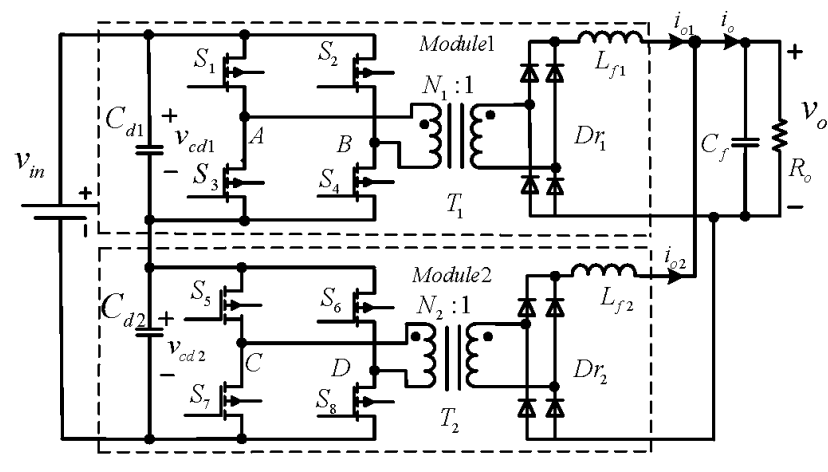

Fig. 1. ISOP connected two FB DC-DC converters.

In practice, these schemes [3],[8]-[16] belong to voltage mode control instead of current mode control. For ISOP converters, direct OCS control results in run-away conditions due to equivalent negative resistance characteristics from each module's input terminals [8],[11]. As a consequence, to achieve a power sharing balance, IVS control loops have to be used in order to achieve excellent power sharing for the ISOP modules. However, current mode control can simplify the design of the outer voltage loop control and improve the power supply performance in many ways, including better dynamics [16].

This paper proposes a digital PS PWM control strategy for ISOP connected two FB DC-DC modules. Moreover, to achieve a power balance between the two modules, an interleaving OCS (IOCS) control strategy is also proposed without any IVS control loops. As a result, the sensing of high input voltages is avoided. Besides, the whole system has many advantages such as simplification of the output voltage regulation (OVR) design. Small signal analysis and loop gain design are also made with the proposed control strategy and a $120 \mathrm{~W}$ prototype is fabricated and experimentally evaluated.

\section{Control Strategy For the System}

Fig. 1 shows the schematic of an ISOP converter made up of two FB DC/DC converters. In this configuration, the total input voltage $v_{i n}$ is divided by the input capacitors $C_{d 1}$ and $C_{d 2}$, thus we obtain the voltages $v_{c d 1}$ and $v_{c d 2}$ working as individual input voltages for each module respectively.

\section{A. Digital PS PWM generation}

A Texas Instrument DSP TMS320F2812 is selected to implement the PWM generation and the closed loop control. The PS-PWM gate signals for all of the switches are generated using the Full Compare Unit 1, the Full Compare Unit 2, the Full Compare Unit 3 and the General Purpose Timer 1 working in up/down counting mode, which can be seen from Fig.2, in which the left legs are leading while the right legs are lagging in phase. CMPR1, CMPR2 and CMPR3 are written to compare the registers and are updated once an underflow or period interrupt occurs. The value of the period register T1PR is determined by the preferred switching frequency and the system clock. There are two interrupts. One is a period interrupt and the other is an underflow interrupt. The values

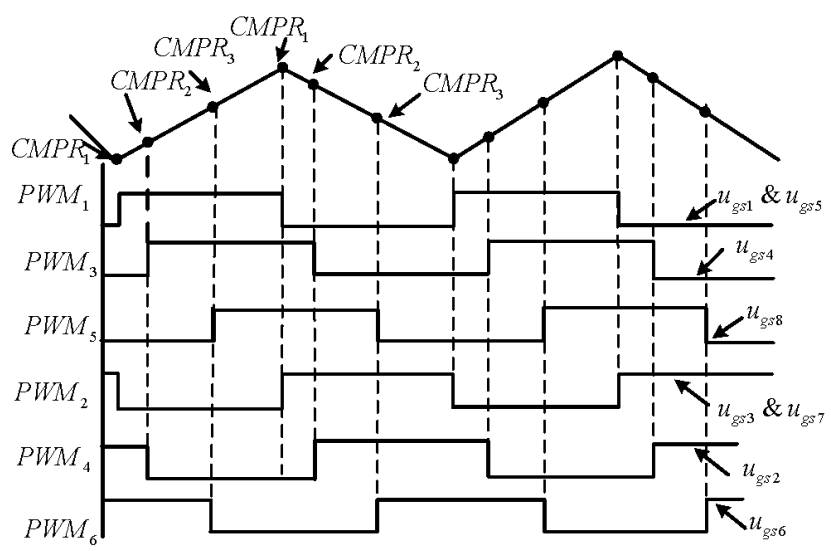

Fig. 2. Schematic of digital PWM generation.

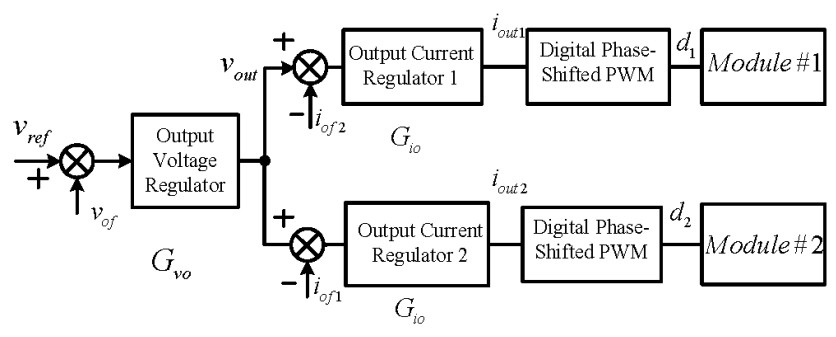

Fig. 3. Proposed control strategy.

of the three compare registers are reloaded and updated at each interrupt, and hence, the three compare matches occur in either the up or down counting mode. In the up counting mode, the compare values are written as:

$C M P R 1=0, C M P R 2=T 1 P R-i_{\text {out } 1}, C M P R 3=T 1 P R-i_{\text {out } 2}$

While in the down counting mode, they are expressed as:

$$
C M P R 1=T 1 P R, C M P R 2=i_{\text {out } 1}, C M P R 3=i_{\text {out } 2}
$$

where $i_{\text {out } 1}$ and $i_{\text {out } 2}$ are the inner current loop outputs of module \#1 and module \#2 respectively, which will be explained later. With the right values set for the three compare registers according to the closed loop regulator output, the six PWM signals are generated as shown in Fig. 2 when the compare match is set high effective. Besides, the PWM signals distribution principle can also be seen. The top switches $\left(\mathrm{S}_{1} \& \mathrm{~S}_{5}\right)$ of the left legs are driven with $\mathrm{PWM}_{1}$ while the bottom switches of the left legs $\left(S_{3} \& S_{7}\right)$ are driven with $\mathrm{PWM}_{2} . \mathrm{S}_{2}$ and $\mathrm{S}_{4}$ of the right top legs are driven with $\mathrm{PWM}_{4}$ and $\mathrm{PWM}_{3}$ respectively while $\mathrm{S}_{6}$ and $\mathrm{S}_{8}$ of the right bottom leg are driven with $\mathrm{PWM}_{6}$ and $\mathrm{PWM}_{5}$ respectively. Note that actually, there is a dead time band between the gate signals for arbitrary legs.

\section{B. Interleaving OCS control}

Fig. 3 illustrates the proposed interleaving OCS control, where a common output voltage regulator provides the current reference $v_{\text {out }}$ for both of the inner current loops. It should be noted that the inner current loop feedbacks are interleaving. This can be explained as follows, the current feedback for 


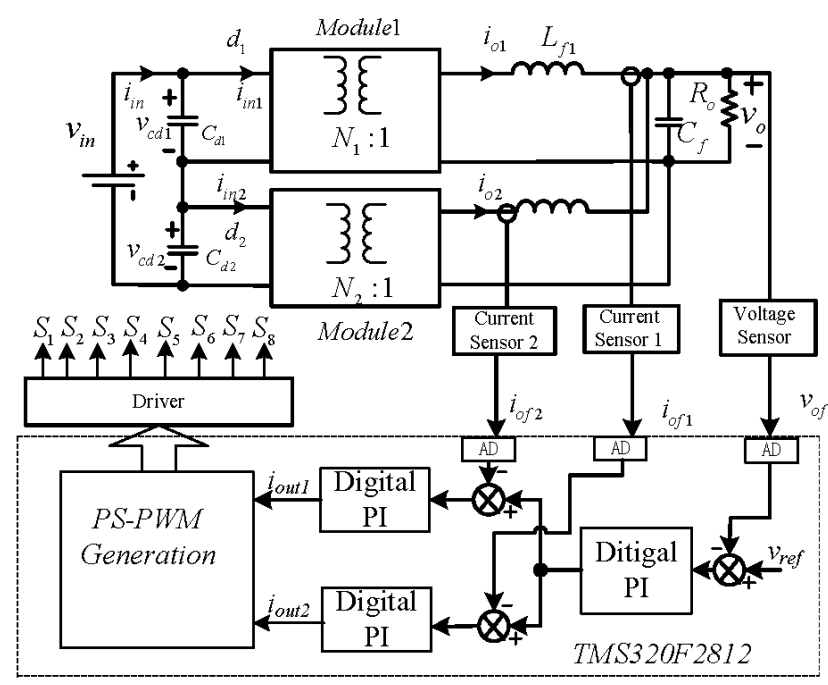

Fig. 4. Block diagram of the digital control system.

one module is the output current of another module while its own output current works as the current feedback of another module. Through compensation of the output current regulator $G_{i o}$, we can derive the inner current loop outputs $i_{\text {out } 1}$ and $i_{\text {out } 2}$ which determine the duty ratios $d_{1}$ and $d_{2}$ respectively.

The stability of the proposed control scheme can be seen as follows. Assuming that the input voltage $v_{\text {in }}$ is constant and that the system works under the steady state, if the input voltage $v_{c d 1}$ increases due to a disturbance, then the input voltage $v_{c d 2}$ decreases, and the output current $i_{o 1}$ increases while the output current $i_{o 2}$ decreases. Based on the control scheme shown in Fig. 3, the duty ratio $d_{1}$ increases while the duty ratio $d_{2}$ decreases. This increases the average input current drawn by module \#1, resulting in a decrease of the input voltage $v_{c d 1}$ and an increase of the input voltage $v_{c d 2}$ until, in the end, equal sharing of the input voltage $v_{\text {in }}$ can be obtained again. On the other hand, when $v_{c d 1}$ decreases due to a disturbance, equal sharing of the input voltage and the load current can be obtained. In the steady state, both of the output currents $i_{\text {out } 1}$ and $i_{\text {out } 2}$ track the OVR output $v_{\text {out }}$ with negligible errors. Therefore, we can derive:

$$
v_{\text {out }}=i_{\text {of } 1}=i_{\text {of } 2} \text {. }
$$

Since the sensing gains of the two output currents are the same, we have $i_{o f 1}=K_{i} i_{o 1}$ and $i_{o f 2}=K_{i} i_{o 2}$, where $K_{i}$ is the sensing gains of the current sensors. According to (3), under the steady state, OCS can be achieved.

The digital control diagram for a ISOP configuration comprising of two modules is shown in Fig.4. The whole control is implemented with a DSP TMS320F2812. The output voltage and the output currents of the individual modules are sampled by an internal AD of the DSP. By interleaving the two output currents as shown in Fig.3 and through digital proportionintegral (PI) compensation, $i_{\text {out } 1}$ and $i_{\text {out } 2}$ which determine the duty cycles for the two modules respectively are obtained. Based on the two values and the digital PWM generation shown in Fig.2, all the gate signals for the two PSFB DC/DC converters are generated.
TABLE I

SYSTEM SPECIFICATIONS

\begin{tabular}{|l|l|l|l|}
\hline \multicolumn{1}{|c|}{ Item } & \multicolumn{1}{c|}{ Value } & \multicolumn{1}{c|}{ Item } & Value \\
\hline Input voltage $v_{i n}$ & $700 \mathrm{~V}$ & Inductance $L_{r}$ & $60 \mu \mathrm{H}$ \\
\hline Output voltage $v_{o}$ & $12 \mathrm{~V}$ & Frequency $f_{s}$ & $50 \mathrm{kHz}$ \\
\hline Load $R_{o}$ & $1.2 \Omega$ & Inductance $L_{f}$ & $0.1 \mathrm{mH}$ \\
\hline Turns ratio $N_{1} 1: 1$ & $4: 1$ & Capacitor $C_{f}$ & $1.0 \mathrm{mF}$ \\
\hline Turns ratio $N_{2}: 1$ & $8: 1$ & $C_{d 1}, C_{d 2}$ & $10 \mu F$ \\
\hline
\end{tabular}
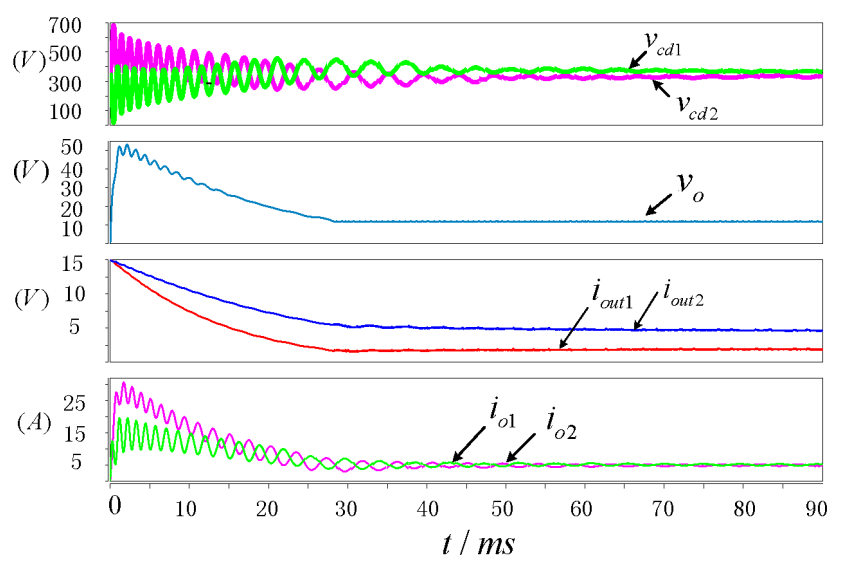

Fig. 5. Simulation waveforms of two-module ISOP system.

For an ISOP connected two full bridge DC-DC converter, a simulation is carried out to verify the effectiveness of the proposed control strategy. The simulation parameters are shown in Table I. As illustrated in the Table, the turns ratios of the two transformers are intentionally made different, with 8:1 for transformer $T_{1}$ and $4: 1$ for transformer $T_{2}$.

Fig. 5 shows the simulation results for the two-module ISOP converter. As seen, during the initial state of the starting process, both the OVR and the current loop outputs saturate. As a result, both modules work under maximum duty cycles, causing unbalanced sharing of the input voltage due to their different turns ratios. Once the output voltage and output currents increase, both the OVR and the inner current controllers come out of saturation, and both the input voltage and the load current are eventually evenly shared under the steady state despite turns-ratio mismatches.

\section{Small Signal Analysis and Regular Loop Gain DESIGN}

\section{A. Small signal modeling of ISOP connected two PS StateDC-} DC converters

Based on the small signal model of PS-FB DC-DC converters, we can derive the small signal model of a two-module ISOP system, as shown in Fig. 6 , where $\hat{d}_{1}$ and $\hat{d}_{2}$ are the perturbations of the duty ratios $d_{1}$ and $d_{2}, v_{c d i}(i=1,2)$ and $i_{o i}(i=1,2)$ represent perturbations of individual input voltages and output currents, and $d_{i j}(j=1,2)$ and $d_{v j}(j=1,2)$ denote perturbations due to output currents and input voltages, which are expressed as:

$$
\left\{\begin{array}{l}
\hat{d}_{i j}=\frac{8 L_{r} f_{s}}{N V_{i n}} \hat{i}_{o j,(j=1,2)} \\
\hat{d}_{v j}=\frac{4 L_{r} D_{e} f_{s}}{N^{2} V_{i n} R_{o}} \hat{v}_{c d j,(j=1,2)}
\end{array}\right.
$$




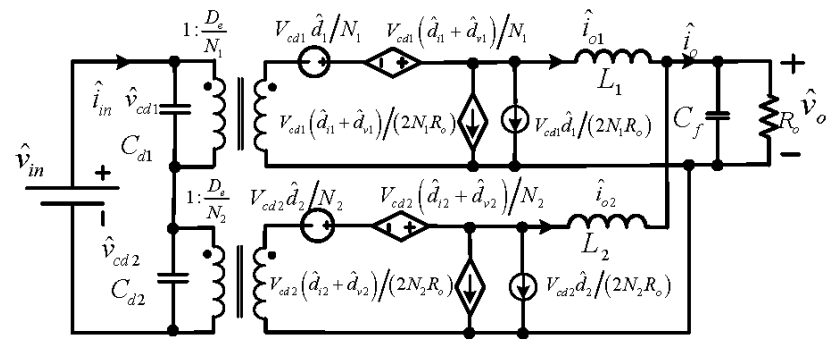

Fig. 6. Small-signal average model of the converter.

For simplicity of analysis, the two modules are assumed to have the same turns-ratio and the same output inductance, i.e., $N_{1}=N_{2}=N$ and $L_{1}=L_{2}=L$. Under the steady state, we have $V_{c d 1}=V_{c d 2}=v_{i n} / 2$. According to Fig.6, we obtain:

$\left\{\begin{array}{l}\frac{D_{e}}{N_{1}} \hat{v}_{c d 1}+\frac{V_{i n}}{2 N}\left(\hat{d}_{1}+\hat{d}_{i 1}+\hat{d}_{v 1}\right)=s L \cdot \hat{i}_{o 1}+\frac{R_{o}}{R_{o} C_{f} s+1}\left(\hat{i}_{o 1}+\hat{i}_{o 2}\right) \\ \frac{D_{e}}{N_{2}} \hat{v}_{c d 2}+\frac{V_{i n}}{2 N}\left(\hat{d}_{2}+\hat{d}_{i 2}+\hat{d}_{v 2}\right)=s L \cdot \hat{i}_{o 2}+\frac{R_{o}}{R_{o} C_{f} s+1}\left(\hat{i}_{o 1}+\hat{i}_{o 2}\right)\end{array}\right.$

Substituting (4) into (5a) and (5b), and then setting $\hat{v}_{c d i}$ $(i=1,2)$, we obtain:

$$
\begin{gathered}
\left(s L+\frac{R_{o}}{R_{o} C_{f} s+1}-\frac{4 L_{r} f_{s}}{N^{2}}\right) \hat{i}_{o 1}+\frac{R_{o}}{R_{o} C_{f} s+1} \hat{i}_{o 2}=\frac{V_{i n}}{2 N} \hat{d}_{1} \\
\frac{R_{o}}{R_{o} C_{f} s+1} \hat{i}_{o 1}+\left(s L+\frac{R_{o}}{R_{o} C_{f} s+1}-\frac{4 L_{r} f_{s}}{N^{2}}\right) \hat{i}_{o 2}=\frac{V_{i n}}{2 N} \hat{d}_{2} .
\end{gathered}
$$

According to (6a) and (6b), we derive:

$$
\left[A^{2}-\left(\frac{R_{o}}{R_{o} C_{f} s+1}\right)^{2}\right] \hat{i}_{o 1}=\frac{V_{i n}}{2 N} \hat{d}_{1} \cdot A-\frac{V_{i n}}{2 N} \hat{d}_{2} \frac{R_{o}}{R_{o} C_{f} s+1}
$$

where:

$$
A=s L+\frac{R_{o}}{R_{o} C_{f} s+1}-\frac{4 L_{r} f_{s}}{N^{2}} .
$$

From (7), we have the transfer functions of the control-tooutput current $\hat{i}_{o i}$ :

$$
\left\{\begin{array}{l}
G_{11}=\left.\frac{\hat{i}_{o 1}}{\hat{d}_{1}}\right|_{\hat{d} 2=0}=\frac{A V_{i n}}{2 N\left[A^{2}-\left(\frac{R_{o}}{R_{o} C_{f} s+1}\right)^{2}\right]} \\
G_{12}=\left.\frac{\hat{i}_{o 1}}{\hat{d}_{2}}\right|_{\hat{d} 1=0}=\frac{-R_{o} V_{i n}}{2 N\left(R_{o} C_{f} s+1\right)\left[A^{2}-\left(\frac{R_{o}}{R_{o} C_{f} s+1}\right)^{2}\right]} .
\end{array}\right.
$$

Likewise, we can obtain the transfer functions of the control-to-output current $\hat{i}_{o 2}$. They are identical to those of the control-to-output current $\hat{i}_{o 1}$, as can be seen from the following:

$$
G_{22}=G_{11}, G_{21}=G_{12} ; .
$$

Hence, the output currents can be written in terms of the controls $\hat{d}_{1}$ and $\hat{d}_{2}$ in the following matrix:

$$
\left[\begin{array}{c}
\hat{i}_{o 1} \\
\hat{i}_{o 2}
\end{array}\right]=\left[\begin{array}{ll}
G_{11} & G_{12} \\
G_{21} & G_{22}
\end{array}\right]\left[\begin{array}{l}
\hat{d}_{1} \\
\hat{d_{2}}
\end{array}\right]=\left[\begin{array}{ll}
G_{m} & G_{n} \\
G_{n} & G_{m}
\end{array}\right]\left[\begin{array}{c}
\hat{d}_{1} \\
\hat{d}_{2}
\end{array}\right] .
$$

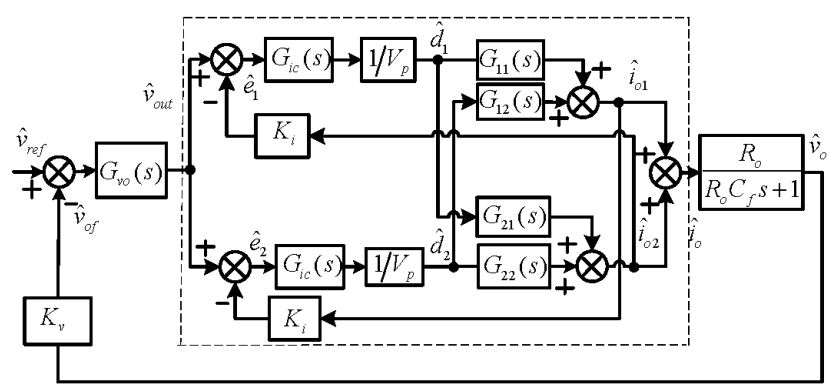

Fig. 7. System block diagram the system with IOCS control.

Based on (11), a closed-loop diagram of the system with the proposed control strategy is illustrated in Fig.7, where $K_{v}$ and $K_{i}$ are the sensor gains of the voltage and the current sensors respectively. $G_{v o}(s)$ is the voltage compensator while the compensators of both inner current loops are identical and denoted as $G_{i c}(s) . V_{p}$ is the peak value of the ramp working as a carrier waveform. Then, the plant transfer function of the inner current loop for module \#1 can be written as:

$$
G_{i d 1}(s)=\left.\frac{\hat{i}_{o 2}}{\hat{d}_{1}}\right|_{\hat{d} 2=0}=G_{21}(s)=G_{n}(s) .
$$

Similarly, the plant transfer function of the inner current loop for module \#2 is expressed as:

$$
G_{i d 2}(s)=\left.\frac{\hat{i}_{o 1}}{\hat{d}_{2}}\right|_{\hat{d} 1=0}=G_{12}(s)=G_{n}(s) .
$$

\section{B. Regulator loop design}

Therefore, the loop gain of each inner current loop can be written as:

$$
T_{i d}(s)=K_{i} G_{i c}(s) G_{n}(s) / V_{p} .
$$

According to the system diagram, the output currents can be written as:

$$
\left\{\begin{array}{l}
\hat{i}_{o 1}=\frac{G_{m}(s) G_{\text {ic }}(s)\left(\hat{v}_{\text {out }}-K_{i} \hat{i}_{o 2}\right)}{V_{p}}+\frac{G_{n}(s) G_{\text {ic }}(s)\left(\hat{v}_{\text {out }}-K_{i} \hat{i}_{\text {o1 }}\right)}{V_{p}} \\
\hat{i}_{\text {o2 }}=\frac{G_{m}(s) G_{\text {ic }}(s)\left(\hat{v}_{\text {out }}-K_{i} \hat{i}_{o 1}\right)}{V_{p}}+\frac{G_{n}(s) G_{\text {ic }}(s)\left(\hat{v}_{\text {out }}-K_{i} \hat{i}_{o 2}\right)}{V_{p}} .
\end{array}\right.
$$

Add the expressions (5a) and (15b), because we have $\hat{i}_{o}=$ $\hat{i}_{o 1}+\hat{i}_{o 2}$. Thus, the equivalent transfer function shown within the dashed box is given by:

$$
G_{\text {iovout }}=\frac{\hat{i}_{o}}{\hat{v}_{\text {out }}}=\frac{2 G_{i c}(s)\left(G_{m}(s)+G_{n}(s)\right)}{V_{p}+G_{m}(s) G_{i c}(s) K_{i}+G_{n}(s) G_{i c}(s) K_{i}} .
$$

Thereby, the loop gain of the OVR loop is expressed by:

$$
T_{v o}(s)=K_{v} G_{v o}(s) G_{\text {iovout }}(s) R_{o} /\left(R_{o} C_{f} s+1\right) .
$$

The specifications of the system are the same with those of the simulation except for the turns ratio. Both of the two transformers have the same turns ratios with $4: 1$. The voltage sensor gain $K_{v}$ and the current sensor gain $K_{i}$ are 0.1 and 0.05 respectively and the ramp amplitude $V_{p}$ is $6 \mathrm{~V}$.

Both the proportional-integral (PI) type compensations for the output voltage and the inner current loops are made. While 


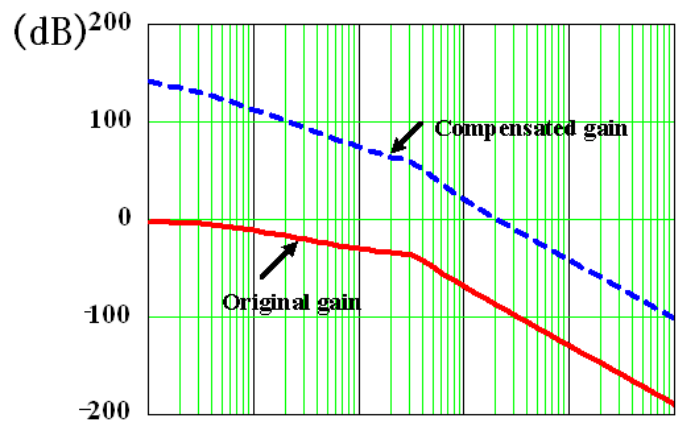

$\left({ }^{\circ}\right)^{200}$

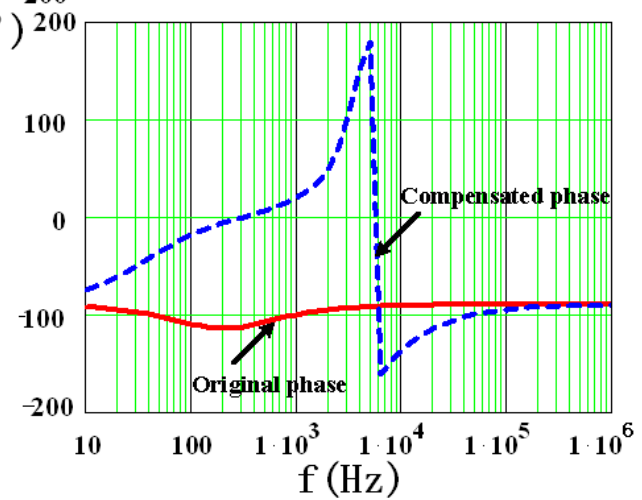

Fig. 8. Uncompensated and compensated OCS loop gain with the IOCS control.
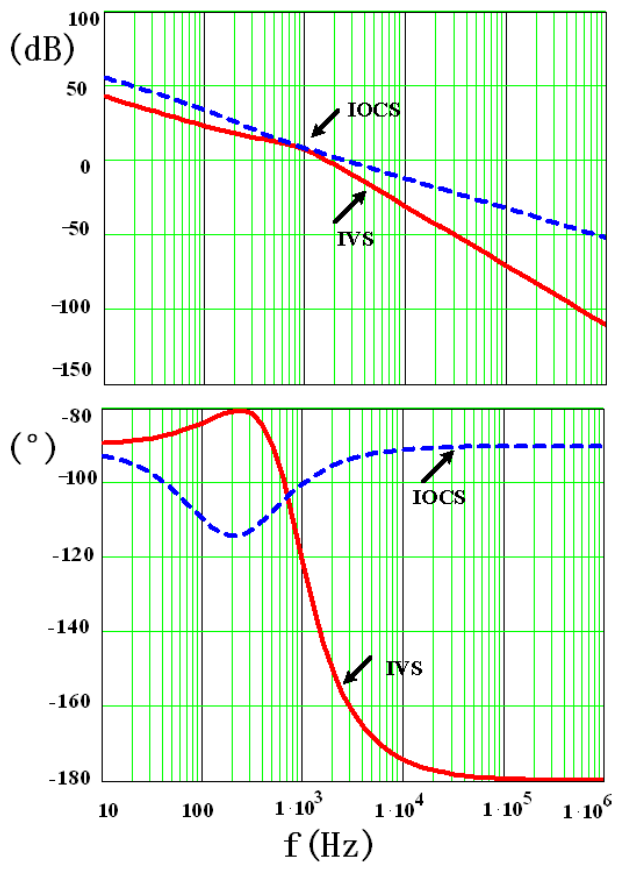

Fig. 9. Compensated OVR loop gains with IVS and IOCS.

under uncompensated conditions, both the transfer functions of the compensators $G_{i c}(s)$ and $G_{v o}(s)$ equal 1 . The crossover frequency of the current loop is chosen to be $20 \mathrm{kHz}$, which is below the half switching frequency. The compensator of the inner current loop is shown as follows:

$$
G_{i c}(s)=10+\frac{25000}{S} \text {. }
$$

As illustrated in Fig.8, the original loop gain of $T_{i d}(s)$ has a magnitude of $-88.5 \mathrm{~dB}$ at $20 \mathrm{kHz}$. However, after compen-

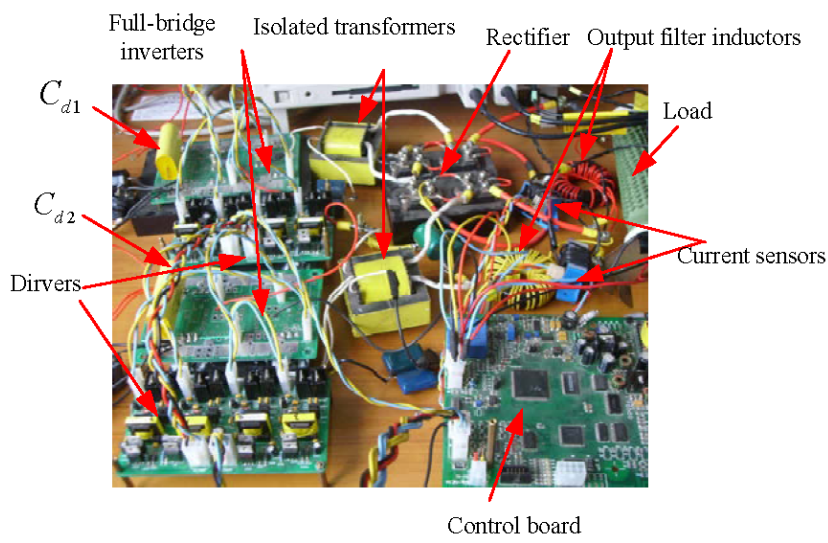

Fig. 10. Prototype of the two phase-shift full-bridge.

sation, at the crossover frequency, the compensated loop gain has a phase margin of $65.57^{\circ}$, which is sufficient for stability.

For the OVR loop gain design, the compensator is written as:

$$
G_{v o}(s)=4+\frac{8000}{S} \text {. }
$$

Fig.9 shows the compensated OVR loop gains with conventional IVS and the proposed control schemes based on the same voltage compensator shown in (18). As can be seen, while in the low frequency region, the magnitude of the loop gain with the IOCS control is larger than that with the IVS control. This implies that the output voltage can track the reference with fewer static errors. Moreover, the compensated loop gains of the OVR with the IOCS control strategy has a crossover frequency of $2.55 \mathrm{kHz}$ with a phase margin of $85.94 \mathrm{o}$ which is sufficiently large to make the whole system stable. However, with the conventional IVS control, the compensated loop has a crossover frequency of $1.67 \mathrm{kHz}$ with a phase margin of $35.49 \mathrm{o}$ which is not large enough to guarantee stability. Because the crossover frequency of the OVR loop with the IVS control is lower than that with the proposed control scheme, the system with the proposed scheme has better dynamic performance. Otherwise, to achieve stability with the IVS control, the compensator function as shown in (18) has to be modified with lower values of the proportion and integration coefficients, but this will result in a decrease of the crossover frequency, leading to further deterioration of the dynamic performance.

\section{EXPERIMENTAL RESULTS}

Experiments on a prototype with the same parameters as those for simulation are made. The system specifications are shown in Table I. The prototype is shown in Fig.10. However, it should be noted that to verify the effectiveness of the proposed control scheme in the presence of mismatches in the converter parameters, the turns ratios of the two power transformers are intentionally designed to be different. The turns ratio of transformer $T_{1}$ is $4: 1$, while turns ratio of $T_{2}$ is $8: 1$.

Fig.11 shows gate signals with the proposed digital PSPWM generation method under its rated input voltage and its rated load. Because the turns ratio of transformer $T_{1}$ is 


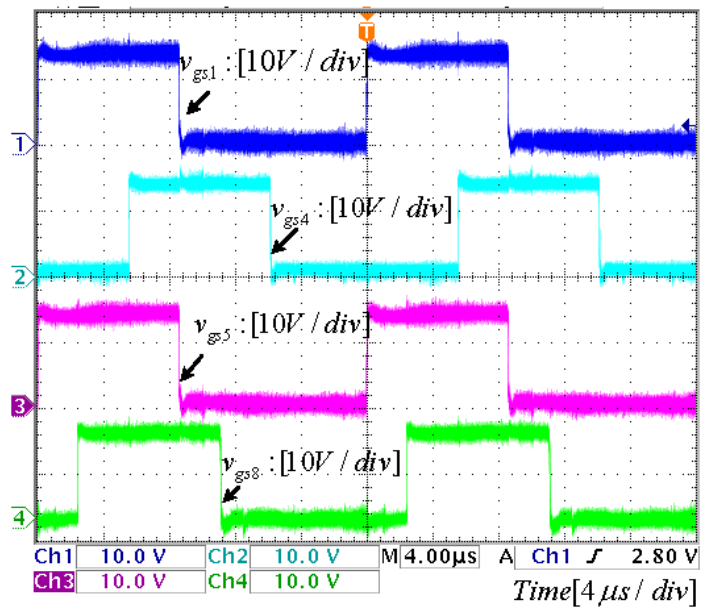

Fig. 11. Generation of digital PS PWM signals.

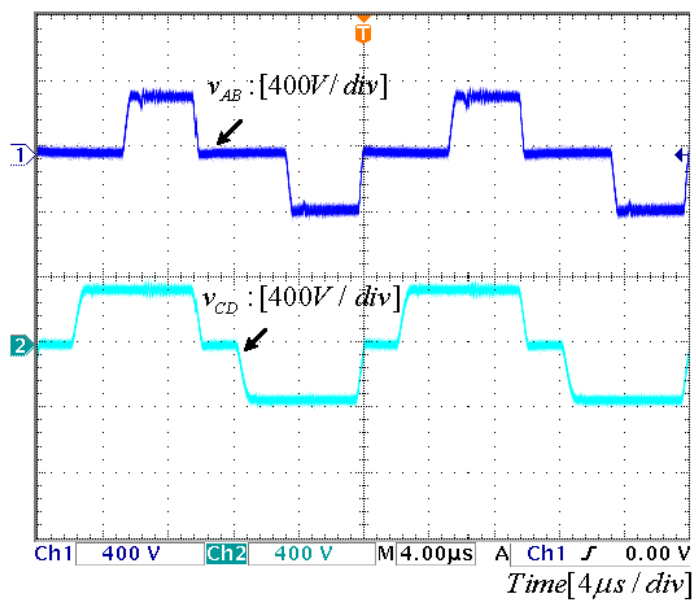

Fig. 12. Transformer primary voltages.

less than that of transformer $T_{2}$, the phase shifted angle of module \#1 is therefore smaller than that of module \#2. Fig.12 illustrates the primary voltages of the power transformers in the system with the gate signals shown in Fig.11. Their amplitudes reveal the corresponding individual input voltages resulting from achieving excellent IVS. Fig.13 illustrates the individual input voltages corresponding to a step change in the total input voltage varying between $600 \mathrm{~V}$ and $800 \mathrm{~V}$. As seen, before and after the transient, the input voltage can be shared equally between the two modules and the output voltage is almost unaffected. Fig.14 shows the individual input voltages corresponding to a load stepping between half $\operatorname{load}(5 \mathrm{~A})$ and full load(10A). Despites the transients, the total input voltage can be shared equally fairly well.

\section{CONCLUSIONS}

In this paper, a method of generating digital PS PWM gating signals for ISOP connected full bridge DC-DC converters is presented.

This paper also proposes an IOCS control strategy for achieving power balance between the ISOP connected two DC-DC modules without using any input voltage control loops. With this scheme, excellent sharing with IVS and

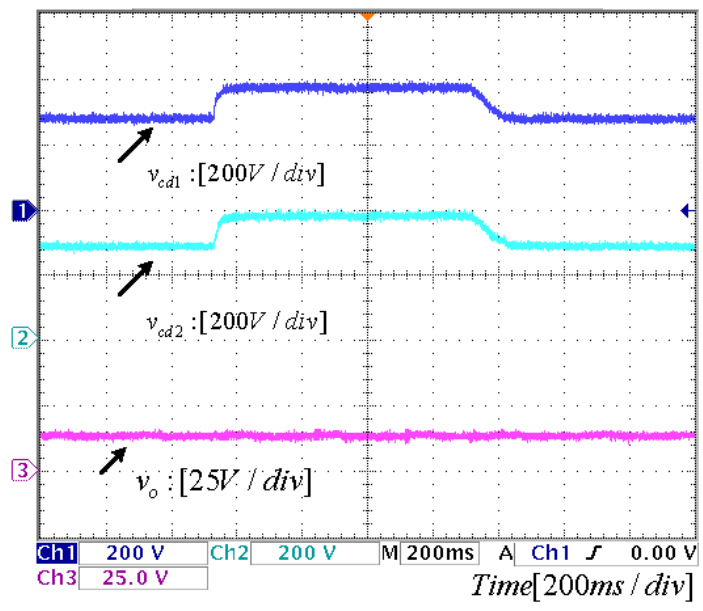

Fig. 13. Response to a step change in total input voltage.

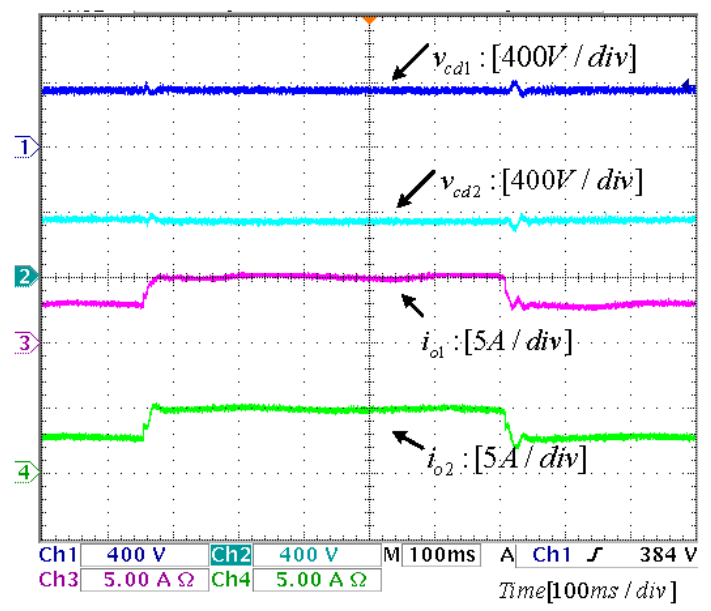

Fig. 14. Response to a step change between full and half load.

OCS can be obtained during the steady state as well as transients in spite of mismatches in the module parameters. In addition, based on small signal analysis and loop gain design for the system, when compared to the widely used IVS control strategies, with the IOCS control, the outer voltage loop design is simplified and the whole system has better static and dynamic performance. A $120 \mathrm{~W}$ prototype based on a TMS320F2812 DSP control has been fabricated and tested, and the experimental results validate the effectiveness of the digital PS-PWM generation method and the IOCS control scheme.

It should be pointed out that the IOCS control strategy can also be applied to other buck-derived ISOP connected two modules by setting the duty cycle losses to zero.

\section{ACKNOWLEDGMENT}

This work was supported by the National Nature Science Foundation of China (Grant No.50807005).

\section{REFERENCES}

[1] F. Canales, P. Barbosa, and F. C. Lee, "A zero-votage and zero-current switching three-level DC/DC conveters," IEEE Trans. Power. Electron. Vol. 17, No. 6, pp. 898-904, Nov. 2002. 
[2] J. A Carr, B. Rowden, and J. C. Balda, "A three-level full-bridge zero-volage zero-current switching converter with a simplified switching sheme," IEEE Trans. Power. Electron., Vol. 24, No. 2, pp.329-338, Feb.2009.

[3] J. W. Kim, J. S. You, and B. H. Cho, "Modeling, control, and design of input-series-output-parallel-connected converter for high-speed-train power system," IEEE Trans. Ind. Electron., Vol. 48, No. 3, pp.534-544, Jun. 2001.

[4] V. Choudhary, E. Ledezma, R. Ayyanar, and R. M. Button ,"Fault tolerant circuit topology and control method for input-series and outputparallel modular DC-DC converters," IEEE Trans. Power. Electron., Vol. 23, No. 1, pp.402-411, Jan. 2008.

[5] D. Sha and X. Liao, "Digital control of switch-mode pulsed welding power," in Proc. Energy Conversion and Congress Exposition, ECCE2009, pp. 2746-2749, San Joes, Sep. 2009.

[6] R. Giri, V. Choudhary, R. Ayyanar and N. Mohan, "Common duty ratio control of input-series connected modular DC-DC converters with active input voltage and load-current sharing," IEEE Trans. Ind. Appl., 2006, Vol. 42, No. 4, pp. 1101-1111, Jul./Aug. 2006.

[7] D. Sha, Z. Guo and X. Liao, "Input series connected high frequency DC DC converters with one transformer," in Proc. Applied Power Electronics Conferences, APEC2010, pp. 662-665, Feb. 2010.

[8] R. Ayyanar, R. Giri, and N. Mohan, "Active input-voltage and loadcurrent sharing in input-series and output-parallel connected modular DC-DC converters using dynamic input-voltage reference sheme," IEEE Trans. Power. Electron., Vol. 19, No. 6,pp.1462-1473, Nov. 2004.

[9] S. P. Natarajan and T. S. Ananadhi, "Control of input series output parallel connected DC-DC converters," Journal of . Power. Electron. Vol. 17, No. 3, pp.265-270, Jul. 2007.

[10] X. Ruan, W. Chen, L. Cheng, C. K. Tse, H. Yan, and T. Zhang,. "Control strategy for input- series- output-parallel converters," IEEE Trans. Ind. Electron., Vol. 56, No. 4, pp. 1174-1185, Apr. 2009,

[11] Y. Huang, C.K. Tse, and X. Ruan, "Gerneral control considerations for input-series connected DC/DC converters," IEEE Trans. Power. Circuit. Syst.I:Reg. Papers, Vol. 56, No. 6, pp.1286-1296, Jun. 2009.

[12] D. Sha, Z. Guo, and X. Liao, "Digital control strategy for input-seriesoutput-parallel modular DC/DC," Journal of Power Electronics, Vol. 10, No. 3, pp. 245-250, May 2010

[13] P. J. Grbovic, "Master/slave control of input-series-and output-parallelconnected converters: concept for low-cost high-voltage auxiliary power supplies," IEEE Trans. Power. Electron., Vol. 24, No. 2,pp.316-328, Feb. 2009.
[14] K. Siri, M. Willhoff, and K. Conner, "Uniform voltage distribution control for series connected DC-DC converters," IEEE Trans. Power Electron., Vol. 22, No. 4, pp. 1269-1279, Jul. 2007.

[15] J. W. Kimball, J. T. Mossoba, and P. T. Krein, "A stabilizing, high performance controller for input-series-output parallel converters," IEEE Trans. Power.Electron., Vol. 23, No. 3, pp.1416-1427, May 2008.

[16] L. Dixon, "Average current mode control of switching power supply," in Proc.Unitrode Power Supply Design Sem.(SEM700), 1990.

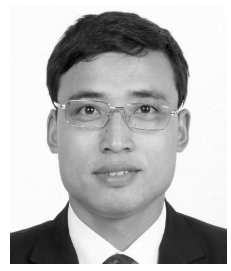

Deshang Sha was born in 1977 . He received his B.S from the Luoyang Institute of Technology, Luoyang, China, in 1998, his M.S. from the Nanjing University of Aeronautics and Astronautics, Nanjing, China in 2001 and his Ph.D. from the Institute of Electrica Engineering, Chinese Academy of Sciences, Beijing, China in 2005, all in Electrical Engineering. From 2005 to 2007, he worked as the Head and Chief Engineer of the full-digitalized welding machine research team of Time Group Company. Since 2008, he has been with the placeSchool of Automation, Beijing Institute of Technology, where he is currently a Lecturer. His research interests are in the areas of digital control of power electronics and renewable energy power generation systems.

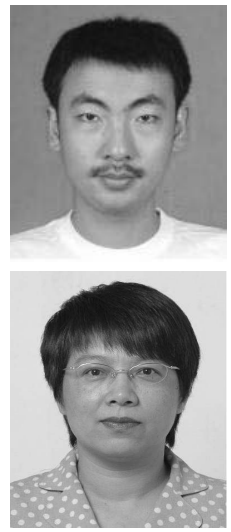

Zhiqiang Guo was born in 1985 . He received his B.S in Automation from the Hebei University of Technology, Tianjin, China, in 2008. He is now pursing his Ph.D. in Electrical Automation at the Beijing Institute of Technology. His research interests are in the areas of modeling and control of power electronics.

Xiaozhong Liao was born in China, in 1962. She received her B.S. and M.S. in Electrical Engineering from Tianjin University, Tianjin, China, in 1982 and 1984 respectively, and her Ph.D. in Control Sciences and Engineering from the Beijing Institute of Technology, Beijing China, in 2004. She was a Visiting Researcher in the Department of Electrical and Electronic Engineering, University of Central Lancashire, Preston, U.K form 1995 to 1996. She is now an Associate Dean and Professor in the placeSchool of Automation, Beijing Institute of Technology. Her research interests are in the fields of power electronics, motor drives and renewable energy power conversion. 